\section{基于国外文献综述的社区环境 儿童出行安全评价指标 \\ EVALUATION INDICATORS OF CHILDREN'S MOBILITY SAFETY IN THE COMMUNITY ENVIRONMENT BASED ON ENGLISH LITERATURE REVIEW}

\section{1 引言}

全球各地在城市化进程中都不可避免地出现了多种社会危机，而 中国的城镇化发展具有规模更大、速度更快的特点 ${ }^{[1]}$, 其与儿童发展 之间的矛盾更加突出。大量高层住宅与商业建筑的密集开发致使公共 游戏场所、绿地系统等锐减，挤压了儿童活动空间; 信息化的快速推

https://doi.org/10.15302/J-LAF-1-020026 收稿时间 RECEIVED DATE / 2020-04-07

中图分类号 / TU984

文献标识码 /

徐梦一

湖南大学建筑学院城乡规划系博士研究生, 西南民族大学讲师

沈瑶

日本千叶大学环境造园学博士, 湖南大学建筑学院城乡规划系副 教授

\section{廖堉轨}

湖南大学建筑学院城乡规划系硕士研究生

海伦·伍和

谢菲尔德大学景观设计系副教授

\section{太通讯作者}

地址：湖南省长沙市岳枆区麓山南路湖南大学

邮编: 410000

那箱: shenya081@hotmai1.com

摘要

联合国儿童基金会的“儿童友好型城市倡 议”以维护儿童权利为核心, 旨在推进有益于儿 童发展的城市及社区环境建设, 而“安全”是最 基本的儿童权利。本文以儿童友好型城市倡议中 社会、健康、出行三大层面的安全要求为基础, 重点探讨如何在社区环境层面评价儿童的出行安 全。研究从41篇国外指标类文献 (含18项评价工 具 ) 和 82 篇其他类文献中提取出评价社区环境下 儿童出行安全的相关指标, 构建含有“机动车交 通环境” “步行/骑行环境” “其他指标”三项 一级指标的评价指标框架, 并根据出行安全的不 同维度，将其进一步分为11项二级指标、29项三 级指标，以及若干四级指标。这一基于国外语境 的出行安全指标框架虽然有待在地性验证，但将 有助于对中国现有环境建设规范中出行安全的相 关要求进行系统性完善、建立更为精细化的分层 指标体系，为项目实践中的各阶段评估应用提供 有益参考。

\section{关键词}

儿童友好型城市；出行安全；社区环境；步行与骑 行; 评价指标; 文献综述

编辑王息瑜田晓劼翻译 当木 王息瑜田晓劼

EDITED BY WANG YinyU TIAN Xiaojie TRANSLATED BY XIAO Jie WANG YinyU TIAN Xiaojie
XU Mengy

PhD Student at Department of Urban and Rural Planning, School of Architecture, Hunan University; Lecturer at Southwest Minzu University

SHEN Yao

Doctor of Environmental Gardening at Chiba University; Associated Professor at Department of Urban and Rural Planning, School Architecture, Hunan University

LIAO Yuhui

Master Student at Department of Urban and Rural Planning, School of Architecture, Hunan University

Helen WOOLLEY

Associated Professor at Department of Landscape Architecture, The University of Sheffield

\section{ABSTRACT}

The Child Friendly Cities Initiative launched by UNICEF aims to protect child rights and promote the establishment of urban and community environment conductive to children development, and safety must be guaranteed first as the precondition of the child rights. Based on social, health, and mobility safety required by the initiative this paper focuses on how to evaluate children's mobility safety in the community environment. After literature review, 41 indicator articles involving 18 assessment tools and 82 other articles were screened, and safety-related indicators were selected to establish an evaluation indicator framework composed of three first-level indictors, i.e., motor traffic environment, walking / bicycling environment, and other indicators. They were further subdivided into 11 second-level indicators, 29 third-level indicators, and more fourth-level indicators. Although this framework needs localized verification and adaption in Chinese cities, it can help systematically improve the mobility safety requirements of the existing regulations and guidelines of urban environment construction to establish a multi-leveled indicator system and provide references for performance evaluation on related practice at all stages.

\section{KEYWORDS}

Child Friendly City; Mobility Safety; Community Environment; Walking and Bicycling; Evaluation Indicators; Literature Review 
(1) 《儿童安全步行十年 倡导报告》显示, 2005 2012年间, 中国 私家车数量与 $0 \sim 14$ 岁 儿童人口数量之比从 1 8.5增至 $1: 2$, 增幅达 2.25 倍。

According to the Ten-Year Report on Children's Safe Walking, from 2005 to 2012 the ratio betwee the amount of private cars and children's population aged below 14 in China increased from $1: 8.5$ to $1: 2$ by a growth of 2.25 -times.
进导致电子媒体改变了儿童游戏生态；城市空间的扩张促使交通基础 设施建设量陡增，而私家车保有量的高增长率 ${ }^{1}$ 不仅加剧了机动车对路 权的绝对控制，还引发了空气污染等一系列公共健康危机，不利于儿 童的安全和健康成长; 越来越多的中国城市儿童受到肥胖症、视力下 降、社交障碍等问题的困扰 ${ }^{[2]}$ 。城镇化带来了城市建成环境的改变，也 加剧了儿童群体在环境健康与卫生、安全保护，以及参与方面所面临 的制约和挑战 ${ }^{[3]}$ 。其中，“安全” 作为最基本的儿童权利，必须首先得 到强力保障

\section{2 儿童友好型城市倡议下的儿童安全}

为了应对上述问题, 共同创建安全的、能够充分响应儿童需求的 包容性城市和社区，联合国儿童基金会 (UNICEF) 和联合国人类住区 规划署于1996年联合发起“儿童友好型城市倡议”（ CFCI），并在随 后的 20 余年中陆续发布了一系列官方文件以指导具体行动的实施。本 研究围绕 “儿童安全”这一基本议题，对官方文件中的纲领性内容进 行了梳理，发现CFCI框架下的儿童安全相关内容包括三个方面：社会 安全、健康安全, 以及出行安全。社会安全是指儿童免于受到剥削、 暴力和虐待, 以及其他违法犯罪行为的威胁; 健康安全是指为儿童提 供清洁、无污染、有绿化的环境, 必需的卫生设施、安全食品、医疗 服务等；而出行安全通常与 “独立出行”一词相关联（表1），侧重于 交通方面的安全。其中, 与城市规划设计学科密切相关的《儿童友好 型城市规划手册：为孩子营造美好城市》在强调独立出行目标的基础 上, 还提出将 “步行友好性” 作为儿童友好型城市 ( CFC) 的环境特征 之一。

儿童安全的三个方面相辅相成, 其最终目的均在于为实现儿童 的基本权利一一生存权、发展权、被保护权和参与权一一提供基础保 障，使儿童能够平等地参与社会事务、享受城市提供的服务与机会，

\section{Introduction}

Globally, various social crises have inevitably emerged along with urbanization, especially in China where this process occurs faster in a larger scale ${ }^{[1]}$, imposing huge threats on children's development. The massive construction of high-density and high-rise residence and commercial buildings results in a sharp reduction of children's activity space such as public playgrounds and green spaces. Booming information technologies have largely influenced children's play with overwhelming electronic media. The urban sprawl results in a surge of transportation infrastructure and a growing number of private cars ${ }^{\mathbb{1}}$, increasing the dominance of motor vehicles over urban traffic and triggering a series of public health crises (such as air pollution), which harm the safety and health of the children. In addition, an increasing number of children in Chinese cities are suffering from obesity, blurred vision, impaired social interaction, etc. ${ }^{[2]}$ All of the above manifest that the changing urban built environment has brought more constraints on environment health and hygiene, security, and participation of children ${ }^{[3]}$. In response, children's safety must be guaranteed as the first child right.

\section{Children Safety under the Child Friendly Cities Initiative}

To address the issues above, UNICEF and UN-Habitat colaunched the Child Friendly Cities Initiative (CFCI) in 1996 and have released a series of guidance documents for building safe and inclusive cities and communities for children. Focusing on the safety issue, this paper reviewed these documents and concluded that children's safety under the CFCI framework involves three aspects: social safety, health safety, and mobility safety. Social safety requires to protect children from exploitation, violence, abuse, and other crimes; health safety is to provide clean, unpolluted, and green environments, and necessary health facilities, safe food and medical services; and mobility safety, often correlated with "independent mobility" (Table 1), focuses on traffic safety. In terms of urban planning and design, the Shaping Urbanization for Children: A Handbook on Child-Responsive Urban Planning further proposes

"walkability" as one of the environmental characteristics of the Child Friendly Cities (CFC).

The three aspects of children's safety together contribute to providing fundamental guarantee for the basic child rights, i.e., the rights to survive, develop, be protected, and participate, allowing children to involve in social affairs on an equal footing and enjoy the services and opportunities in the city for 
(2) 本文中的社区环境是 指在一定行政区划范 围内的社区户外公共 空间环境。

\begin{tabular}{|c|c|}
\hline $\begin{array}{l}\text { 文件名称 } \\
\text { File }\end{array}$ & $\begin{array}{l}\text { 与儿童 “出行安全” 相关的内容 } \\
\text { Contents about children's mobility safety }\end{array}$ \\
\hline $\begin{array}{l}\text { 《儿童友好型城市规划手册》 }{ }^{[6]} \\
\text { Shaping Urbanization for Children: } \\
\text { A Handbook on Child-Responsive } \\
\text { Urban Planning }\end{array}$ & $\begin{array}{l}\text { 提出10项儿童权利与城市规划原则，其中第5条原则为: 为儿童和社区发展积极出行和公共交通, 确 } \\
\text { 他们能独立出行, 让他们有平等、安全的渠道获取城市提供的所有服务和机会。 } \\
\text { Adopting } 10 \text { Children's Rights and Urban Planning Principles, and Principle } 5 \text { is about } \\
\text { Transportation Systems - Develop active transportation and public transit systems and ensure } \\
\text { independent mobility for children and the community, so they have equal and safe access to all } \\
\text { services and opportunities in their city. } \\
\text { 提出7个儿童友好型城市环境特征, 包括特征3 “可步行” 以及特征6 “独立出行” 。 } \\
\text { Proposing seven conceptual standards of child-friendly urban environments, including Standard 3, } \\
\text { "walkability" and Standard 6, "independent mobility." }\end{array}$ \\
\hline $\begin{array}{l}\text { 《构建儿童友好城市和社区手册》占] } \\
\text { UNICEF Child Friendly Cities and } \\
\text { Communities Handbook }\end{array}$ & $\begin{array}{l}\text { 附件6 “儿童友好型城市倡议的变化理论” 提出的 } 20 \text { 个具体目标之一: 为儿童独立出行提供更完善的 } \\
\text { Annex VI "CFCI Theory of Change" comes up with } 20 \text { objectives, and one of them is "Improved } \\
\text { children's independent mobility." }\end{array}$ \\
\hline $\begin{array}{l}\text { 《创建儿童友好城市行动框架》 } \\
\text { Building Child Friendly Cities: } \\
\text { A Framework for Action }{ }^{[6]}\end{array}$ & $\begin{array}{l}\text { 提出CFC必须保障儿童的12项权利, 其中第7项为 “能够安全、单独地在街道上行走 (的权利) ” } \\
\text { Proposing that CFC guarantees } 12 \text { rights of every young citizen, and the seventh is "walk safely in } \\
\text { the streets on their own." }\end{array}$ \\
\hline $\begin{array}{l}\text { 《儿童友好度社区自测工具包》 } \\
\text { Child Friendly Community Self- } \\
\text { Assessment Tool for Children }{ }^{[7]}\end{array}$ & $\begin{array}{l}\text { “安全与保护” 板块的测评内容包括 “儿童行走及骑车的安全” 以及 “乘坐公交车和其他公共交通 } \\
\text { 的安全感” } \\
\text { In the "My Safety \& Protection" test section, there are yes / no questions "it is safe for me to walk } \\
\text { and cycle in my community" and "I feel safe using buses or other public vehicles." }\end{array}$ \\
\hline
\end{tabular}

注

CFCI更关注与儿童体力活动相关的积极出行活动的安全, 而公共交通安全在各类文件中仅侧重于提高儿童的“安全感” , 因此本研究未将公共 交通安全纳入考虑范围。

NOTE

$\mathrm{CFCl}$ calls for a greater attention to the active mobility safety related to the children's physical activities. Public transportation safety focuses on

the improvement of the "sense of safety" and is therefore excluded from this paper.
(2) The community environment in this paper refers to the outdoor public space of a community in an administrative area
并得到全面发展。其中，“出行安全”与建成环境设计的关联度最 高, 故本文将基于国外文献对其评价指标展开研究。

\section{3 社区环境儿童出行安全评价指标研究概要}

\section{1 研究对象}

家庭、学校、社会是儿童成长的三大环境，而社区环境 ${ }^{2}$ 在其中起 着重要的连接作用, 承载着户外游戏、出行、社交等诸多儿童日常行 为活动, 故本研究聚焦于社区层面的儿童出行安全。

\section{2 研究方法及目的}

本文采用文献综述的研究方法，对与 “社区环境” 相关的关 键词进行归类检索，并对获取到的有效文献中的出行安全相关指 comprehensive development. In the consideration of the close relation between mobility safety and the built environment, this paper focuses on the evaluation indicators of children's mobility safety based on English literature review.

\section{Research Synopsis of the Evaluation Indicators on the Community Environment for Children's Mobility Safety}

\subsection{Research Object}

Family, school, and society are the major environments where children grow up. They are bonded by the community environment $^{2}$, where most children's daily activities (such as outdoor playing, traveling and social activities) occur. This paper thus focuses on children's mobility safety at the community level.

\subsection{Research Methods and Purpose}

Based on a review on literature relevant to "community environment," this paper retrieved indicators of mobility safety 
标进行提取和梳理, 形成社区环境儿童出行安全指标集。其中, 有 效文献分为：1）指标类文献：主题词中包含 “指标” 相关中心词 ( “index” “indicator” “measurement” 等), 通常针对特定的社区环 境评价工具, 可从中直接获取与出行安全相关的指标内容；2）其他类 文献：此类文献的主题词不包含 “指标” 相关中心词, 但可根据其研 究结果判断从评价工具中提取的安全指标是否合理, 以及补充评价工 具未包含但与本研究相关的其他指标。研究目的在于通过构建基于国 外相关文献的 “社区环境儿童出行安全” 评价指标框架, 为中国开展 此类评价指标的研究及应用提供有价值的参考。

\section{4 建立评价指标框架}

\section{1 文献检索过程及结果}

本次文献检索主要基于ScienceDirect期刊数据库，同时辅以谢菲尔 德大学StarPlus资源库, 以及 Web of Science数据库, 并将检索结果分为 “指标类文献” 及 “其他类文献” 进行进一步研读。

研究首先选取 “child” ( 儿童)、“safety”（安全）、 “community”（社区）、“road”（道路）、“walk”（步行）、 “mobility” (出行) 等作为基本关键词。由于环境的 “步行友好性” (walkability) 常被用于衡量社区对体力活动以及积极出行相关内容的 支持程度 ${ }^{[8][9]}$, 因此将 “walk” 的扩展关键词 “walkability” (步行友好 性）、“walkable” (可步行)、“walking / bicycling suitability”（步 行/骑行适宜性) 等也纳人检索范围。此外, 通学行为是儿童最重要的 日常活动之一, 而父母对安全因素的考虑往往对儿童能否独立通学以 及通学方式的选择有重要影响, 因此 “CIM” (Children's Independent Mobility，儿童独立活动性）“travel to school”（上学）以及 “school commute” (通学) 等相关词语也被纳人检索范围。研究最终共确定 了 44 个检索关键词, 分为出行行为及其属性、评价环境、面向群体、 “指标” 相关中心词 4 个类别 (表 2 )，每次选取 $2 \sim 3$ 个不同类别的关 键词, 进行多次组合检索。检索结果篮选原则如下： 1) 以2009 2020 年间发表的文献为主, 结果不足时可追溯; 2) 必须是经过同行审议的 学术类期刊的英文文献。

根据上述检索步骤及原则, 最终获取有效文献162篇, 其中指标类 51篇（包含24项评价工具）, 其他文献111篇。经过阅读文献, 筛选出 可提取出行安全指标的文献共计 123 篇, 其中指标类 41 篇, 涉及 18 项评 in the valid literature, and came up with an indicator set for children's mobility safety in community environment. The valid literature includes: 1 ) indicator literature: the articles whose topic words include indicator-related core words (index, indicator, measurement, etc.), generally focusing on a certain assessment tool for the community environment and directly providing evaluation indicators about mobility safety; and 2) other literature: the articles do not include any indicatorrelated topic word but can be used to judge if the indicators retrieved from the assessment tools are valid or not, and to supplement indicators not included in the assessment tools but related to the study. The purpose of this paper is to establish an evaluation indicator framework of children's mobility safety in the community environment among English literature, thus providing references for research and application of such evaluation indicators in China.

\section{Establishment of the Evaluation Indicator Framework}

\subsection{Literature Retrieval Process and Results}

The literature retrieval was mainly conducted on ScienceDirect, supplemented by StarPlus of University of Sheffield and Web of Science. The results were divided into indicator literature and other literature .

First, "child," "safety," "community," "road," "walk," and "mobility," etc. were selected as the basic keywords. Second, considering that "walkability" is often used to measure a community's supporting level to physical activities and active $\operatorname{travel}^{[8][9]}$, "walkability," "walkable," and "walking / bicycling suitability" were also listed as keywords. School commute is one of the most important activities in children's daily life, but whether and how they can do this independently are highly determined by parents' safety considerations. Therefore, keywords such as "CIM" (Children's Independent Mobility), "travel to school," and "school commute" were also added. Finally, a total of 44 keywords in four categories (mobility behavior and behavioral attributes, environment assessed, target group, and indicator-related core words) were identified (Table 2). In each retrieval, 2 or 3 keywords from different categories were used together, and the valid articles were screened if they 1) were published between 2009 and 2020, when there was no enough search results, articles published earlier could be included; and 2) were peer-reviewed and published in English academic journals.

Finally, a total of 162 valid articles were obtained, 51 of which were indicator articles (including 24 assessment tools) and 111 were other articles. Then they were all examined and 


\begin{tabular}{|c|c|}
\hline $\begin{array}{c}\text { 类别 } \\
\text { Category }\end{array}$ & $\begin{array}{c}\text { 关键词 } \\
\text { Keywords }\end{array}$ \\
\hline $\begin{array}{l}\text { 出行行为及其属性 } \\
\text { Mobility behaviors and their } \\
\text { attributes }\end{array}$ & $\begin{array}{l}\text { Walk / walkability / walking suitability / bicycling / cycling } \\
\text { mobility / independent mobility / CIM / } \\
\text { travel to school / school commute / traffic / transport / activity }\end{array}$ \\
\hline $\begin{array}{l}\text { 评价环境 } \\
\text { Environment assessed }\end{array}$ & $\begin{array}{l}\text { Neighborhood / community / environment } \\
\text { road / street / route / pavement / sidewalk/ walkways / path } \\
\text { park / recreation space / recreational space / green space / play } \\
\text { space / public space / outdoor }\end{array}$ \\
\hline $\begin{array}{l}\text { 面向群体 } \\
\text { Target group }\end{array}$ & Child / kid / youth / teens / adolescent / pedestrian \\
\hline $\begin{array}{l}\text { “指标” 相关中心词 } \\
\text { Core words related to "indicator" }\end{array}$ & $\begin{array}{l}\text { Assessment / audit / tool / evaluation / index } \\
\text { criteria / measurement / indicator / instrument }\end{array}$ \\
\hline
\end{tabular}

价工具, 评价内容主要包括道路安全、步行/骑行等活动及其环境, 以 及其他儿童出行目的地三个类别; 其他类文献82篇（图1）。

\subsection{8 项评价工具中出行安全相关指标研究}

本研究篮选出的18项评价工具基本信息汇总如表 3 所示。所有工具 皆由欧美学者制定，其中14项来自美国，其余4项分别来源于英国、澳 大利亚、芬兰和加拿大; 发布时间跨度为1998 2017年, 其中W ABSA 发布时间最早，除2007年没有发布任何评价工具、2002年及2015年各发 布两项以外，其他均匀分布于2003 2017年间，每年发布一项。评价 工具中提及儿童群体或包含儿童群体相关具体指标的有 14 项, 其中完 全面向儿童群体的仅有NEWS-Y、MAPS、EWI\&MI, 以及PARK4项。

文献检索与筛选结果

1. Classify and screen literature retrieval results
123 of them were kept for collecting evaluation indicators on mobility safety, 41 of which were indicator articles involving 18 assessment tools for road safety, walking / bicycling behaviors and environments, and other destinations of children mobility; and 82 were other articles (Fig. 1).

\subsection{Evaluation Indicators for Mobility Safety in 18 Assessment Tools}

Table 3 lists the basic information of the 18 assessment tools, 14 of which are from the United States and the other 4 are from Britain, Australia, Finland, and Canada. WABSA, the earliest tool, was published in 1998; from then on there was one tool published each year till 2017, except 2007 (none was published), 2002 and 2005 (two were published respectively). Children and indicators for them are mentioned in 14 tools, among which only NEWS-Y, MAPS, EWI \& MI, and PARK focus on

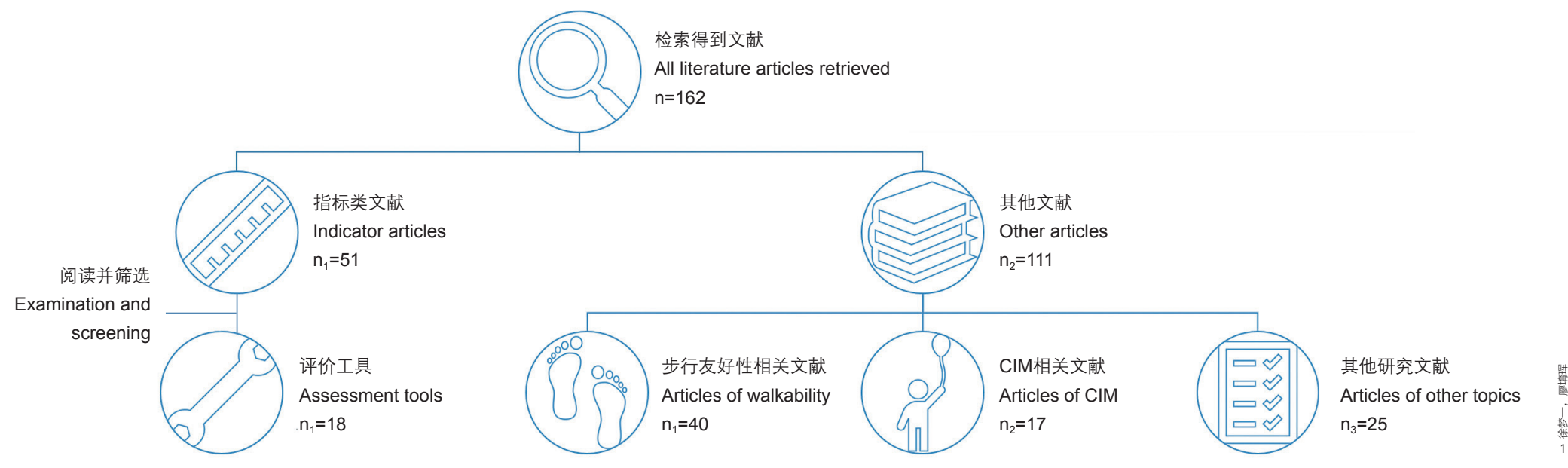




\begin{tabular}{|c|c|c|c|c|c|c|c|c|}
\hline \multirow{2}{*}{$\begin{array}{l}\text { 评价工具名称 } \\
\text { Name of tool }\end{array}$} & \multirow{2}{*}{$\begin{array}{c}\text { 研究者 } \\
\text { Researchers }\end{array}$} & \multirow{2}{*}{$\begin{array}{l}\text { 发布时间 } \\
\text { Year of } \\
\text { publishing }\end{array}$} & \multirow{2}{*}{$\begin{array}{l}\text { 国家 } \\
\text { Country }\end{array}$} & \multirow{2}{*}{$\begin{array}{l}\text { 面向人群 } \\
\text { Target group }\end{array}$} & \multirow{2}{*}{$\begin{array}{l}\text { 评价目的 } \\
\text { Purpose }\end{array}$} & \multicolumn{3}{|c|}{$\begin{array}{c}\text { 评价环境类型 } \\
\text { Type of environment assessed }\end{array}$} \\
\hline & & & & & & $\begin{array}{l}\text { 机动车交通环境 } \\
\text { Motor traffic } \\
\text { environment }\end{array}$ & $\begin{array}{c}\text { 步行/骑行环境 } \\
\text { Walking / bicycling } \\
\text { environment }\end{array}$ & $\begin{array}{l}\text { 其他儿童户外活动环境 } \\
\text { Other environments for } \\
\text { children's outdoor activities }\end{array}$ \\
\hline $\begin{array}{l}\text { 苏格兰步行友好性评价工具 ( SWAT) } \text { ) }^{[10]} \\
\text { Scottish Walkability Assessment Tool } \\
\text { (SWAT) }{ }^{[10]}\end{array}$ & $\begin{array}{l}\text { 凯瑟琳·米林顿等 } \\
\text { Catherine Millington et al. }\end{array}$ & 2008 & $\begin{array}{l}\text { 英国 } \\
\text { UK }\end{array}$ & $\begin{array}{l}\text { 全域龄 } \\
\text { All ages }\end{array}$ & $\begin{array}{l}\text { 揭示环境特征与体力活动的关联性 } \\
\text { To demonstrate the relevance between } \\
\text { environmental characteristics and physical activity }\end{array}$ & & - & \\
\hline $\begin{array}{l}\text { 邻里环境青少年步行友好性度量表 } \\
\text { ( NEWS-Y) } \\
\text { Neighborhood Environment Walkability } \\
\text { Scale for Youth (NEWS-Y) }\end{array}$ & $\begin{array}{l}\text { 多利 } \cdot \text { 罗森伯格等 } \\
\text { Dori Rosenberg et al. }\end{array}$ & 2009 & $\begin{array}{l}\text { 美国 } \\
\text { USA }\end{array}$ & $\begin{array}{l}\text { 12 18岁儿童; } \\
5 \sim 18 \text { 岁儿童的父母 } \\
\text { 12 18 year-old children; } \\
\text { parents of } 5 \text { ～18 year-old } \\
\text { children }\end{array}$ & $\begin{array}{l}\text { 通过主观感受评估环境与体力活动的关系 } \\
\text { To assess the relations between the environment } \\
\text { and physical activity through subjective perception }\end{array}$ & & • & \\
\hline $\begin{array}{l}\text { 步行及骑行环境系统性评估 } \\
\text { ( SPACES) } \\
\text { Systematic Pedestrian and Cycling } \\
\text { Environmental Scan (SPACES) }\end{array}$ & $\begin{array}{l}\text { 泰利 } \cdot J \cdot \text { 皮克拉等 } \\
\text { Terry J. Pikora et al. }\end{array}$ & 2002 & $\begin{array}{l}\text { 澳大利亚 } \\
\text { Australia }\end{array}$ & $\begin{array}{l}\text { 全域龄 ( 提及儿童 ) } \\
\text { All ages (children are } \\
\text { specially mentioned) }\end{array}$ & $\begin{array}{l}\text { 测量影响社区内步行和骑行活动的物理环境因素 } \\
\text { To measure physical environmental factors affecting } \\
\text { walking and cycling in the community }\end{array}$ & & $\bullet$ & \\
\hline $\begin{array}{l}\text { 微观步行环境量表 (MAPS) } \text { ) }^{[13]} \\
\text { Microscale Audit of Pedestrian } \\
\text { Streetscapes (MAPS) }{ }^{[13]}\end{array}$ & $\begin{array}{l}\text { 詹姆斯 } \cdot F \cdot \text { 塞利斯等 } \\
\text { James F. Sallis et al. }\end{array}$ & 2015 & $\begin{array}{l}\text { 美国 } \\
\text { USA }\end{array}$ & $\begin{array}{l}\text { 6 16岁儿童 } \\
\text { 6 16 year-old children }\end{array}$ & $\begin{array}{l}\text { 探究体力活动与微观尺度环境具体指标的关联度 } \\
\text { To measure the correlation between physical activity } \\
\text { and specific indexes of micro-scale environment }\end{array}$ & & • & \\
\hline $\begin{array}{l}\text { 步行与骑行可持续性评估 ( WABSA) }{ }^{[14]} \\
\text { Walkability and Bicycling Suitability } \\
\text { Assessment (WABSA) }{ }^{[14]}\end{array}$ & $\begin{array}{l}\text { 詹姆斯 } \cdot F \cdot \text { 塞利斯等 } \\
\text { James F. Sallis et al. }\end{array}$ & 1998 & $\begin{array}{l}\text { 美国 } \\
\text { USA }\end{array}$ & $\begin{array}{l}\text { 全域龄 ( 提及儿童 ) } \\
\text { All ages (children are } \\
\text { specially mentioned) }\end{array}$ & $\begin{array}{l}\text { 评估城市街道的步行及骑行友好性 } \\
\text { To assess the walkability and cyclability of urban } \\
\text { streets }\end{array}$ & & - & \\
\hline $\begin{array}{l}\text { 道路环境量表 ( PEAT ) }{ }^{[15]} \\
\text { Path Environment Audit Tool (PEAT) }\end{array}$ & $\begin{array}{l}\text { 菲利普·J · 特罗培德等 } \\
\text { Philip J. Troped et al. }\end{array}$ & 2005 & $\begin{array}{l}\text { 美国 } \\
\text { USA }\end{array}$ & $\begin{array}{l}\text { 全域龄 ( 提及儿童 ) } \\
\text { All ages (children are } \\
\text { specially mentioned) }\end{array}$ & $\begin{array}{l}\text { 从物理特性、设计、设施和美观/维护 } 4 \text { 个维度评估社 } \\
\text { 区步道 } \\
\text { To assess community trails from four dimensions: } \\
\text { physical characteristics, design, facilities, and } \\
\text { aesthetics / maintenance }\end{array}$ & & $\bullet$ & \\
\hline $\begin{array}{l}\text { 步行环境指标测度 ( PEDS ) 工具 }{ }^{[16]} \\
\text { Pededstrian Environmental Data Scan } \\
\text { (PEDS) Tool }{ }^{\left[{ }^{[6]}\right.}\end{array}$ & $\begin{array}{l}\text { 凯利 } \cdot \text { J · 克里芬等 } \\
\text { Kelly J. Clifton et al. }\end{array}$ & 2007 & $\begin{array}{l}\text { 美国 } \\
\text { USA }\end{array}$ & $\begin{array}{l}\text { 全域龄 } \\
\text { All ages }\end{array}$ & $\begin{array}{l}\text { 步行环境评估 } \\
\text { To assess walking environment }\end{array}$ & & $\bullet$ & \\
\hline $\begin{array}{l}\text { 环境对体力活动的支持度问卷 } \\
\text { ( ESPAQ ) } \\
\text { Env] } \\
\text { Activity Quentionnaire (ESPAQ) }{ }^{[17]}\end{array}$ & $\begin{array}{l}\text { 芭芭拉 } \cdot \text { E } \cdot \text { 安斯沃斯等 } \\
\text { Barbara E. Ainsworth et al. }\end{array}$ & 2002 & $\begin{array}{l}\text { 美国 } \\
\text { USA }\end{array}$ & $\begin{array}{l}\text { 全域龄 } \\
\text { All ages }\end{array}$ & $\begin{array}{l}\text { 以问卷形式评估环境对体力活动的支持度 } \\
\text { To assess environmental support for physical } \\
\text { activity with questionnaire }\end{array}$ & & • & \\
\hline $\begin{array}{l}\text { 积极生活社区清单 ( ANC ) }{ }^{[18]} \\
\text { Active Neighborhood Checklist (ANC) })^{[18]}\end{array}$ & $\begin{array}{l}\text { 克里斯汀 } \cdot M \cdot \text { 赫纳等 } \\
\text { Christine } M . \text { Hoehner et al. }\end{array}$ & 2007 & $\begin{array}{l}\text { 美国 } \\
\text { USA }\end{array}$ & $\begin{array}{l}\text { 全域龄 ( 提及儿童 ) } \\
\text { All ages (children are } \\
\text { specially mentioned) }\end{array}$ & $\begin{array}{l}\text { 从街道尺度评估社区环境中与体力活动行为相关的 } \\
\text { 特征 } \\
\text { To assess the characteristics associated with } \\
\text { physical activities in community environment at } \\
\text { street scale }\end{array}$ & & - & \\
\hline $\begin{array}{l}\text { 社区公园量表 (CPAT) })^{[19]} \\
\text { Community Park Audit Tool (CPAT) }\end{array}$ & $\begin{array}{l}\text { 安德鲁· } \mathrm{T} \cdot \text { 卡钦斯基等 } \\
\text { Andrew T. Kaczynski et al. }\end{array}$ & 2010 & $\begin{array}{l}\text { 美国 } \\
\text { USA }\end{array}$ & $\begin{array}{l}\text { 全域龄 ( 提及儿童 ) } \\
\text { All ages (children are } \\
\text { specially mentioned) }\end{array}$ & $\begin{array}{l}\text { 评估社区公园对于体力活动的支持水平 } \\
\text { To assess the support level of community park for } \\
\text { physical activity }\end{array}$ & & & • \\
\hline $\begin{array}{l}\text { 公共游息空间环境评价工具 ( EAPRS) } \text { ) }^{[20 \mid 2121]} \\
\text { Environmental Assessment of Public } \\
\text { Recreation Spaces Tool (EAPRS })^{200 \mid 121]}\end{array}$ & $\begin{array}{l}\text { 布莱恩 } \cdot \mathrm{E} \cdot \text { 西伦斯等 } \\
\text { Brian E.Saelens et al. }\end{array}$ & 2006 & $\begin{array}{l}\text { 美国 } \\
\text { USA }\end{array}$ & $\begin{array}{l}\text { 全域龄 ( 提及儿童 ) } \\
\text { All ages (children are } \\
\text { specially mentioned) }\end{array}$ & $\begin{array}{l}\text { 公共游螅空间环境综合评价 } \\
\text { Commrehensive evaluation of public recreational } \\
\text { space }\end{array}$ & & & - \\
\hline $\begin{array}{l}\text { 道路安全评估（TARVA） } \\
\text { TARVA }\end{array}$ & $\begin{array}{l}\text { 哈利·佩尔托拉等 } \\
\text { Harri Peltola et al. }\end{array}$ & 2012 & $\begin{array}{l}\text { 芬兰 } \\
\text { Finland }\end{array}$ & $\begin{array}{l}\text { 全域龄 } \\
\text { All ages }\end{array}$ & $\begin{array}{l}\text { 道路安全综合评估 } \\
\text { Comprehensive assessment of road safety }\end{array}$ & $\bullet$ & & \\
\hline $\begin{array}{l}\text { 步行评分工具 (WS ) } \text { [ }^{[23]} \\
\text { Walking Score (WS) }\end{array}$ & $\begin{array}{l}\text { 弗里曼·兰斯等 } \\
\text { Freeman Lance et al. }\end{array}$ & 2017 & $\begin{array}{l}\text { 美国 } \\
\text { USA }\end{array}$ & $\begin{array}{l}\text { 全域龄 ( 提及儿童 ) } \\
\text { All ages (children are } \\
\text { specially mentioned) }\end{array}$ & $\begin{array}{l}\text { 步行相关指标评分 } \\
\text { Walking-related index score }\end{array}$ & & • & \\
\hline $\begin{array}{l}\text { 步行友好性性指标 ( WI ) } \\
\text { Walkabiliti I Index (WI) }\end{array}$ & $\begin{array}{l}\text { 布莱恩 } \cdot E \cdot \text { 西伦斯等 } \\
\text { Brian E. Saelens et al. }\end{array}$ & 2003 & $\begin{array}{l}\text { 美国 } \\
\text { USA }\end{array}$ & $\begin{array}{l}\text { 全域龄 ( 提及儿童 ) } \\
\text { All ages (children are } \\
\text { specially mentioned) }\end{array}$ & $\begin{array}{l}\text { 评价可步行性 } \\
\text { To evaluate walkability }\end{array}$ & & $\bullet$ & \\
\hline
\end{tabular}




\begin{tabular}{|c|c|c|c|c|c|c|c|c|}
\hline \multirow{2}{*}{$\begin{array}{l}\text { 评价工具名称 } \\
\text { Name of tool }\end{array}$} & \multirow{2}{*}{$\begin{array}{c}\text { 研究者 } \\
\text { Researchers }\end{array}$} & \multirow{2}{*}{$\begin{array}{l}\text { 发布时间 } \\
\text { Year of } \\
\text { publishing }\end{array}$} & \multirow{2}{*}{$\begin{array}{l}\text { 国家 } \\
\text { Country }\end{array}$} & \multirow{2}{*}{$\begin{array}{c}\text { 面向人群 } \\
\text { Target group }\end{array}$} & \multirow{2}{*}{$\begin{array}{l}\text { 评价目的 } \\
\text { Purpose }\end{array}$} & \multicolumn{3}{|c|}{$\begin{array}{c}\text { 评价环境类型 } \\
\text { Type of environment assessed }\end{array}$} \\
\hline & & & & & & $\begin{array}{c}\text { 机动车交通环境 } \\
\text { Motor traffic } \\
\text { environment }\end{array}$ & $\begin{array}{c}\text { 步行/骑行环境 } \\
\text { Walking / bicycling } \\
\text { environment }\end{array}$ & $\begin{array}{l}\text { 其他儿童户外活动环境 } \\
\text { Other environments for } \\
\text { children's outdoor activities }\end{array}$ \\
\hline $\begin{array}{l}\text { 步行友好性与可移动性指标 } \\
\text { ( EWI\&I) } \\
\text { Environment walkability Index and } \\
\text { Moveability Index (EWI \& MII) }\end{array}$ & $\begin{array}{l}\text { 克里斯托弗·巴克等 } \\
\text { Christoph Buck et al. }\end{array}$ & 2014 & $\begin{array}{l}\text { 美国 } \\
\text { USA }\end{array}$ & $\begin{array}{l}\text { 2 9岁儿童 } \\
\text { 2 9 year-old children }\end{array}$ & $\begin{array}{l}\text { 评价与儿童体力活动（步行友好性及可移动性）相关 } \\
\text { 联的环境指标 } \\
\text { To assess environmental indicators associated with } \\
\text { children's physical activity (walkability and moveability) }\end{array}$ & & • & \\
\hline $\begin{array}{l}\text { 公园、儿童活动及休闲量表 ( PARK) })^{[26]} \\
\text { The Parks, Activity and Recreation } \\
\text { among Kids (PARK) }\end{array}$ & $\begin{array}{l}\text { 玛德琳 } \cdot E \cdot \text { 伯德等 } \\
\text { Madeleine E. Bird et al. }\end{array}$ & 2015 & $\begin{array}{l}\text { 加拿大 } \\
\text { Canada }\end{array}$ & $\begin{array}{l}5 \sim 18 \text { 岁儿童 } \\
5 \sim 18 \text { year-old children }\end{array}$ & $\begin{array}{l}\text { 评估与儿童体力活动相关的公园特征 } \\
\text { To assess park's characteristics associated with } \\
\text { children's physical activity }\end{array}$ & & & $\bullet$ \\
\hline $\begin{array}{l}\text { 青少年公园质量指标 ( QUINPY ) } \\
\text { QUalitity INdex of Parks } \\
\text { for Youth (QUINPY) }\end{array}$ & $\begin{array}{l}\text { 亚历山德罗 · 瑞格伦等 } \\
\text { Alessandro Rigolon et al. }\end{array}$ & 2016 & $\begin{array}{l}\text { 美国 } \\
\text { USA }\end{array}$ & $\begin{array}{l}\text { 全域龄 ( 提及儿童 ) } \\
\text { All ages (children are } \\
\text { specially mentioned) }\end{array}$ & $\begin{array}{l}\text { 从儿童需求及利用角度评估城市公园 } \\
\text { To assess city parks from the perspective of } \\
\text { children's needs and use }\end{array}$ & & & $\bullet$ \\
\hline 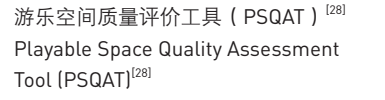 & $\begin{array}{l}\text { 加文 } \cdot R \cdot \text { ·詹金斯等 } \\
\text { Gavin R. Jenkins et al. }\end{array}$ & 2015 & $\begin{array}{l}\text { 美国 } \\
\text { USA }\end{array}$ & $\begin{array}{l}\text { 全域龄 ( 提及儿童 ) } \\
\text { All ages (children are } \\
\text { specially mentioned) }\end{array}$ & $\begin{array}{l}\text { 以游戏价值为切入点, 评估儿童游戏场所 } \\
\text { To evaluate children's play place focusing on game } \\
\text { value }\end{array}$ & & & $\bullet$ \\
\hline \multicolumn{5}{|l|}{$\begin{array}{l}\text { 注 } \\
\text { 1. NEWS-Y度量表分为青少年版本和家长版本 } \\
\text { 2. 研究人员在原MAPS工具 (共150个指标) 基 } \\
\text { 其中以 } 6 \sim 11 \text { 岁儿童为研究对象的NIK量表以 }\end{array}$} & \multicolumn{4}{|c|}{$\begin{array}{l}\text { NOTES } \\
\text { 1. NEWS-Y is published in both adolescent version and parent version. } \\
\text { 2. Based on the original MAPS (with } 150 \text { indicators in total), four MAPS-mini tools were further developed for different age } \\
\text { groups. This study reviewed the NIK (Neighborhood Impact on Kids) tool for children between } 6 \text { and } 11 \text { years old, and } \\
\text { the TEAN (Teen Environment and Neighborhood) tool for children between } 12 \text { and } 16 \text { years old, both with } 15 \text { indicators. }\end{array}$} \\
\hline
\end{tabular}

评价工具涉及三类环境：1）机动车交通环境；2）步行/骑行环境；3） 其他儿童户外活动环境。

18 项评价工具的开发目的、面向人群及所评价的环境各异, 其 中仅有SWAT、NEW-Y、CPAT及WS明确将 “安全” 作为一项独立评 价内容并提出了细分指标, 但未能涵盖 “安全” 问题的所有层面; 其 他工具中的安全相关指标则分散于不同层级的框架下。因此, 现有评 价工具中尚不存在全面的安全专项指标集, 这正凸显了本研究的重要 意义。同时, 各评价工具中对安全类指标的细分程度及分类标准也各 有不同。因此, 本研究将所有安全相关指标进行提取, 并初步总结为 “机动车交通环境” “步行/骑行环境” “其他指标” 三个大类, 依次 包含7个、13个以及 8 个小类 (表4), 为建立层级更清晰的指标框架打 下基础。

总体来看, 没有任何一项评价工具可以覆盖表4所示全部指标小 类。根据表3 4, 虽然本研究中多达 $66.7 \%$ 的评价工具涉及对步行/骑行 the children exclusively. Overall, three types of environment were assessed: motor traffic environment, walking / bicycling environment, and other environments for children's outdoor activities.

The 18 tools differ in the development purpose, target group, and type of environment assessed. Only SWAT, NEW-Y, CPAT and WS assess "safety" separately with detailed indicators, which are still not enough for comprehensive evaluation, while safety indicators in other tools scatter unsystematically. Therefore, there is not a safety indicator set in existing English literature. At the same time, the subdivision and classification standard of the safety indicators vary among all the tools. This study therefore re-organized all safety-related indicators into three categories: motor traffic environment (with 7 sub-category indicators), walking / bicycling environment (with 13 subcategory indicators), and other indicators (with 8 sub-category indicators) (Table 4).

There is no tool covering all sub-category indicators shown in Table 4. In Table 3 and 4, although $66.7 \%$ of the evaluation 
环境的评价，但 “步行/骑行环境” 大类指标的占表率仅为 $38.9 \%$; 涉及

“机动车交通环境” 的评价工具较少，但 “机动车交通环境” 大类指 标的占表率却达到 $21.4 \%$, 因此可认为机动车交通环境对儿童出行安全 有较为重要的影响。“步行/骑行环境” 大类下的各指标小类在不同评 价工具间分布相对均匀（图2）。

根据对各指标小类的分项统计，机动车交通控制、步行/骑行通行 设施、步行/骑行辅助设施、步道/自行车道表面、其他步行/骑行设施 tools have indicators for walking / bicycling environment, the average coverage ratio of the "walking / bicycling environment" category is only $38.9 \%$, while fewer tools involve motor traffic environment assessment but the average coverage ratio of the "motor traffic environment" category is $21.4 \%$. It thus can be concluded that the motor traffic environment has a significant impact on children's mobility safety. The indicators of the "walking / bicycling environment" category appear quite evenly among the assessment tools (Fig. 2).

Among all the tools, motor traffic control, general walking / bicycling facilities, walking / bicycling auxiliary

表4: 18项评价工具中安全相关指标的初步分类

Table 4: Rough categorization of safty-related indicators in the 18 assessment tools

\begin{tabular}{|c|c|c|c|c|c|c|c|c|c|c|c|c|c|c|c|c|c|c|c|c|c|}
\hline \multirow{2}{*}{$\begin{array}{c}\begin{array}{c}\text { 指标大类 } \\
\text { Category }\end{array} \\
\begin{array}{c}\text { 指标小类 } \\
\text { Sub-category }\end{array}\end{array}$} & \multicolumn{7}{|c|}{$\begin{array}{c}\text { 机动车交通环境 } \\
\text { Motor traffic environment }\end{array}$} & \multicolumn{13}{|c|}{$\begin{array}{c}\text { 步行/䐀行环境 } \\
\text { Walking / bicycling environment }\end{array}$} & \multirow{2}{*}{\begin{tabular}{|c} 
其他 \\
Other indicators \\
无人看管的 \\
动物等8项 \\
Others (in total of \\
8 items, including \\
unattended \\
animals)
\end{tabular}} \\
\hline & $\begin{array}{l}\text { 交通控制 } \\
\text { Traffic } \\
\text { control }\end{array}$ & $\begin{array}{l}\text { 辅助设施 } \\
\text { Auxiliary } \\
\text { facilities }\end{array}$ & $\begin{array}{l}\text { 交通量 } \\
\text { Traffic } \\
\text { volume }\end{array}$ & $\begin{array}{c}\text { 交通速度 } \\
\text { Speed }\end{array}$ & $\begin{array}{l}\text { 车道 } \\
\text { Lanes }\end{array}$ & $\begin{array}{c}\text { 弯曲度 } \\
\text { Curvature }\end{array}$ & $\begin{array}{l}\text { 坚向设计 } \\
\text { Vertical } \\
\text { design }\end{array}$ & $\begin{array}{c}\text { 一般通行设施 } \\
\text { General crossing } \\
\text { facilities }\end{array}$ & $\begin{array}{l}\text { 辅助设施 } \\
\text { Auxiliary } \\
\text { facilities }\end{array}$ & $\begin{array}{c}\text { 节点路缘 } \\
\text { Curb }\end{array}$ & $\begin{array}{c}\text { 坡度 } \\
\text { Slope }\end{array}$ & \begin{tabular}{|c|} 
宽度 \\
Width
\end{tabular} & $\mid \begin{array}{c}\text { 连续度 } \\
\text { Continuity }\end{array}$ & $\begin{array}{c}\text { 类型 } \\
\text { Types }\end{array}$ & \begin{tabular}{|c|} 
表面 \\
Surfaces
\end{tabular} & \begin{tabular}{|c|} 
界面 \\
Boundary
\end{tabular} & $\begin{array}{c}\text { 障碍物 } \\
\text { Obstacles }\end{array}$ & $\begin{array}{l}\text { 其他设施 } \\
\text { Other } \\
\text { facilities }\end{array}$ & $\begin{array}{l}\text { 周边监视 } \\
\text { Surrounding } \\
\text { surveillance }\end{array}$ & $\begin{array}{l}\text { 其他视线 } \\
\text { Other } \\
\text { sights }\end{array}$ & \\
\hline SWAT & V & & & & & & & V & V & V & & & & & V & V & & & & & \\
\hline NEWS-Y & & V & V & V & & & & V & $V$ & & & & & & & & & & V & & V \\
\hline SPACES & V & & & & $\checkmark$ & & & V & V & V & V & & V & & V & & V & V & V & & V \\
\hline MAPS & V & & & & V & & & V & V & & V & & & V & V & V & V & V & & & \\
\hline WABSA & & & V & V & $\mathrm{V}$ & & & V & $V$ & V & & & V & & V & V & & V & & V & \\
\hline PEAT & 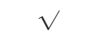 & & & & & & & V & V & V & V & & & V & V & V & V & V & & V & V \\
\hline PEDS & V & & & V & $\mathrm{V}$ & & & V & $v$ & V & V & V & V & V & V & V & V & V & & & \\
\hline ESPAQ & & & V & & & & & & & & & & & & V & & & V & & & V \\
\hline ANC & V & & & & $v$ & & & V & V & V & V & V & V & & V & V & V & V & & & \\
\hline CPAT & V & & & & & & & V & V & & & & & V & V & & & V & V & & V \\
\hline EAPRS & V & V & V & & & V & V & V & & & & V & & $V$ & V & & & V & V & V & V \\
\hline TARVA & V & & & V & & & & V & V & & & & & V & & V & V & V & & V & \\
\hline wS & V & & & & & & & & & & & & & & V & & & & & & V \\
\hline WI & & & & & & & & & & & & & & & & & & & & & V \\
\hline EWI \& MI & & & & & & & & & & & & & & & & & & & & & V \\
\hline PARK & & & & & & & & & & & & & & & & & & V & V & & \\
\hline QUINPY & & & & & & & & & & & & & & & & & & & & & V \\
\hline PSQAT & & & & & & & & & & & & & & & & & & V & V & & V \\
\hline $\begin{array}{c}\text { 指标小类出现次数 } \\
\text { 总计 } \\
\text { Count of indicators } \\
\text { in the } \\
\text { sub-category }\end{array}$ & 10 & 2 & 4 & 4 & 5 & 1 & 1 & 11 & 10 & 6 & 5 & 3 & 4 & 6 & 11 & 7 & 6 & 12 & 6 & 4 & 11 \\
\hline $\begin{array}{c}\text { 指标大类 } \\
\text { 出现次数总计 } \\
\text { Count of } \\
\text { indicators in the } \\
\text { category }\end{array}$ & & & & 27 & & & & & & & & & & 91 & & & & & & & 11 \\
\hline
\end{tabular}




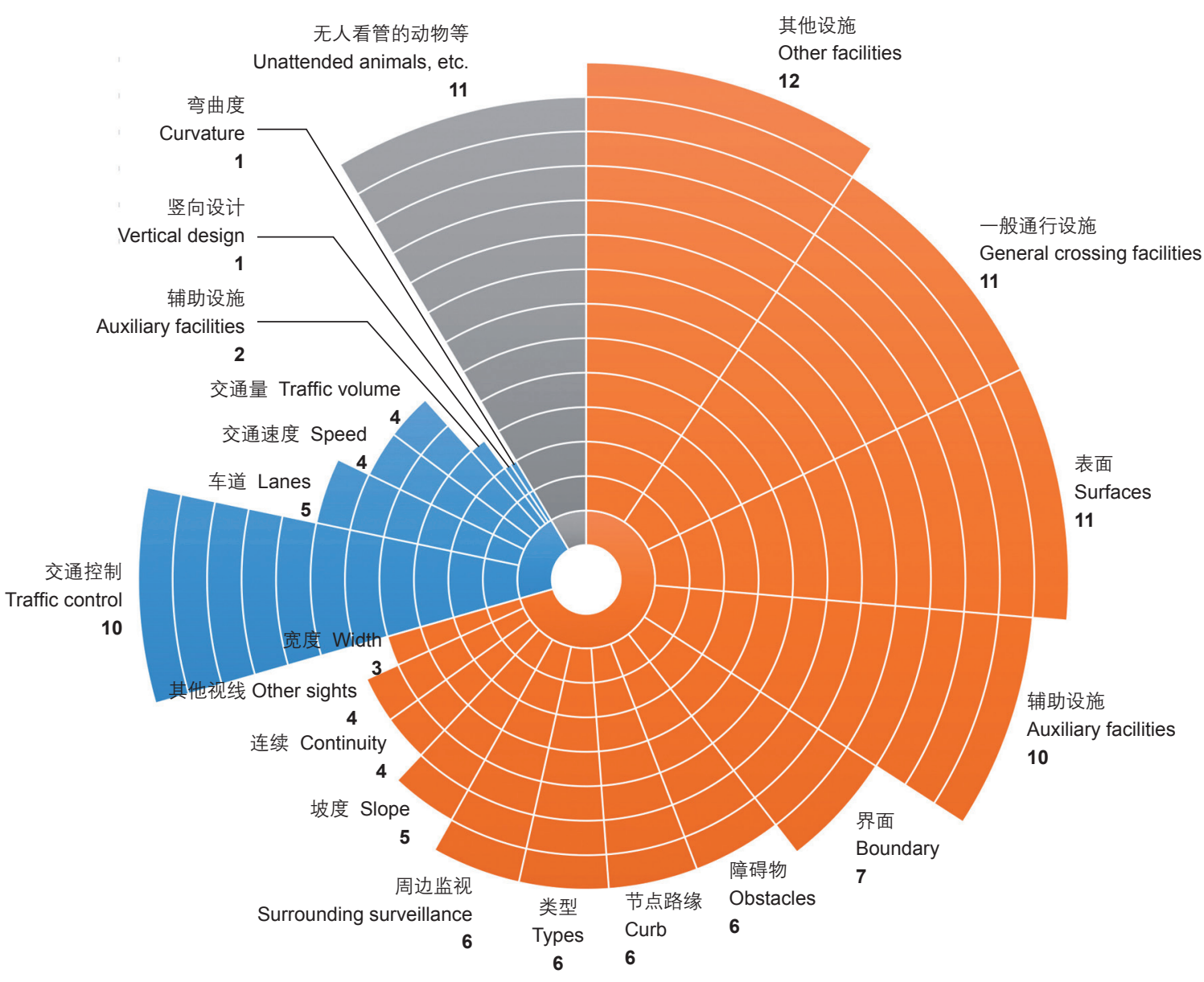

各指标小类分布情况

2. The distribution among indicators of the subcategories
5 类在全部工具中出现次数最多 (不少于 10 次) ; 其次为机动车道情 况, 步道/自行车道节点路缘、坡度、类型和界面, 以及步行/骑行环境 中的障碍物及周边监视 7 类。

另外由表4可知， $61 \%$ 的评价工具涉及 “其他指标” 大类，包括机 动车尾气排放、邻里识别度 (易于寻路) 、噪音、难闻的气味、邻里 信任度熟知度、无人看管的动物等，与 “健康安全” 的评价内容有所 交叉, 有待进一步讨论。

\section{3 其他出行安全文献研究}

在对指标类文献中的 18 项评价工具进行梳理之后，本研究又对 82 篇其他类文献进行了系统性整理。

“步行友好性” 相关文献指出, 邻里环境的步行友好性的一个标 准差变化会导致积极出行增加 $27.5 \%$, 这一点在年轻群体中尤为明显 ${ }^{[5]}$ 。 另有研究从 “安全性” “实用性” “愉悦度”三个方面对步行环境 facilities, footpath / bikeway surfaces, and other walking / bicycling facilities appear the most (at least mentioned in 10 tools). Motor vehicle lanes, and curbs, slope, types, boundary, obstacles, and surrounding surveillance of walking / bicycling environment are the second most mentioned indicators.

According to Table 4, 61\% of the tools have the "other indicators," such as vehicle exhaust, legibility within the neighborhood (easy for wayfinding), noise, odor, neighborhood trust / close-knit, and unattended animals. Some of these indicators are also related to children's health safety, which need further discussion.

\subsection{Study on Other Articles on Mobility Safety}

After reviewing the 18 assessment tools in the indicator literature, this study went through the rest 82 articles.

Research on walkability suggests that one standard deviation change in neighborhood walkability was associated with an estimated $27.5 \%$ increase in active travel trips, which is especially notable among the young ${ }^{[5]}$. Some researchers 
进行评价, 其中 “安全性” 权重达到 0.4 , 为三者中最高 ${ }^{[29]}$ 。因此从步 行友好性评价研究中提取 “安全” 相关指标是有效且有意义的。

CIM 被定义为无父母监管时儿童在邻里环境内的自由活动 ${ }^{[30]}$, 安全 是其最重要的影响因素, 通常包含社会安全与交通安全两类 ${ }^{[311-[33]}$, 有 研究还特别强调步行安全 ${ }^{[3]}$ 。从 CIM 研究文献中也解析出了若干与步行 友好性研究相一致的细化安全指标, 例如交通量、交通速度等 ${ }^{[35][39]}$, 但大多属于 “机动车交通环境” 指标大类，仅有 “交通节点穿行保 障” 等 $[37][39[40]$ 属于 “步行/骑行环境” 指标大类。虽然现有评价工具中 也包括步行道路状况 (如铺装、宽度等) 类指标, 但青少年群体对社 区步行友好性的模糊评价证实, 相较于路面质量, 公共安全与交通安 全对独立出行的影响更大 ${ }^{[4]}$ 。除此之外, 来自陌生人的攻击、骚扰、欺 凌及反社会行为等也是影响儿童独立出行的社会安全因素 ${ }^{[42-[44]}$; “照 明” 则是一种值得探讨的增加夜晚出行安全感知的因素 ${ }^{[39][40]}$, 可被纳 人表4 “步行骑行环境” 下的 “其他设施” 指标小类。值得注意的是, CIM研究对表4中 “其他指标” 类所涉及的环境因子也较为关注, 尤其 从儿童独立出行需求的角度探讨了更为广泛的安全问题, 例如动物产 生的威胁、迷路, 以及熟人或关系良好的邻里一一他们的视线可形成 被动监督, 也可以成为儿童寻求帮助的对象, 从而为独立出行的儿童 带来安全感 ${ }^{[43][44]}$, 而这些因素在其他研究中往往被忽略, 故而对CIM研 究文献的检索有效地扩展了本研究的思路, 有助于进一步完善评价指 标框架。

儿童通学研究也将安全因素作为重点研究内容, 涵盖了诸多社会 及物理环境因素, 如邻里关系、陌生人的威胁、步行设施（包括交通 信号灯、步行过街路口、步道数量等) 、机动车交通量、街道机动车 停放数量, 以及道路连续度等。此外, “区域 (社区) 对道路安全的 重视程度”是一项意识层面的新指标。[45][46]

此外, 虽然驾驶员及行人的不可预测或不当行为 ${ }^{[47 \mid 48]}$ 也会对儿童出 行造成安全威胁, 但主要需通过公共安全教育 ${ }^{[4]}$ 和严格执法 ${ }^{[50]}$ 等非环境 设计手段对其进行干预，故本研究不予以考量。 measured the environment's walkability in terms of safety, practicability, and pleasure, while safety enjoyed the highest weight of $0.4^{[29]}$. Therefore, it is effective and meaningful to select safety-related indicators from walkability evaluation research.

Defined as the children's free activities in the neighborhood without parental supervision ${ }^{[30]}$, CIM is mainly influenced by

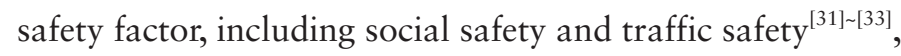
and some CIM studies lay particular emphasis on walking safety ${ }^{[34]}$. Detailed safety indicators in CIM literature, such as traffic volume and speed ${ }^{[35] \sim[39]}$, are also mentioned in walkabilityrelated studies. However, most of them belong to the category of "motor traffic environment" in Table 4, and only a few of them, such as travel supporting at crossing ${ }^{[37[39][40]}$, belong to the "walking / bicycling environment" category. Although the path condition (pavement, width, etc.) are also included as safety indicators in the above assessment tools, a fuzzy evaluation on walkability by adolescents proved that it is less important than public safety and traffic safety when it comes to $\mathrm{CIM}^{[41]}$. In addition, attack, harassment, bullying, and antisocial behaviors of strangers also pose social safety threats to $\mathrm{CIM}^{[42]-[44]}$. Lighting, as an indicator of "other facilities" in "walking / bicycling environment" category, helps increase the safety perception during trips at night ${ }^{[39[400]}$. It is worth noting that CIM studies also attach greater attention to environmental factors related to the "other indicators" category in Table 4, with wider safety concerns such as threats from animals, getting lost, and acquaintance or friendly neighbors who can provide passive surveillance of sight and assistance in children's independent mobility ${ }^{[43][44]}$ - these factors are often overlooked in other studies. Therefore, CIM literature review provides new perspectives to help set up a more comprehensive indicator framework.

School commute studies also attach much importance to safety factors at social and physical-environment aspects, like neighborhood relationship and threats from strangers, walking facilities (including traffic lights, pedestrian crossings, and the amount of footpaths), motor traffic volume, amount of vehicles parked in the street, and continuity of walking paths. The community's concern about road safety is a new indicator at the awareness level. ${ }^{[45][46]}$

In addition, this study does not cover unpredictable behaviors and misconducts of drivers or pedestrians ${ }^{[47][48]}$ which also threaten children's mobility safety potentially, since they need to be addressed with public safety education ${ }^{[49]}$ and strict law enforcement ${ }^{[50]}$ rather than environmental design. 


\section{Conclusions}

\section{1 社区环境儿童出行安全评价指标框架的建立}

综上所述, 本研究提出基于国外文献的社区环境儿童出行安全评 价指标框架，包含一级指标三项，二级指标11项，三级指标29项，四 级指标若干 (表5)。其中, 一级指标中的 “机动车交通环境” 和 “步 行/骑行环境” 主要从机动车交通控制、步行/骑行通行保障、交通节点 穿行保障、视觉保障及提示、其他保障等维度评价儿童的出行安全; “其他指标” 则分为社会安全、感知安全、生理健康和安全意识 4 个 层面。

\section{2 国外指标的局限性与中国指标的在地性思考}

根据上述国外文献综述结果, 可知目前有关儿童出行安全的评价 指标研究总体数量较少。本研究尝试从 4 类关键词人手, 对既有文献进 行挖掘, 发现现有指标研究存在明显的趋同现象, 例如对儿童活动空 间的安全研究大多关注视线内的监督保护、交通连接, 以及局部环境 的安全因素指标 (如照明情况) 等, 而未考虑儿童活动的连续性 ${ }^{3}$, 因 此存在较为明显的局限性。目前虽有一些城市已颁布了相关指导性文 件 (如纽约市为提升街道安全性而出台的《街道设计手册》和《创造 更安全的街道》导则 $\left.{ }^{[5]}\right)$, 也开展了以儿童为主体的多种形式的实践 （如澳大利亚墨尔本市菲利普港的 “绿灯工程” 、意大利的 “我们独 自上学” 计划, 以及美国、澳大利亚、新西兰等国的 “步行校车” 计 划 ${ }^{[52[153]}$ 等)，但仍缺乏对已知安全影响因子的系统性整合，对其可靠性 及实践效果的评估也不足。

此外, 本文所述文献及指标工具均非针对中国语境, 其文化背 景、社区情况、所针对的儿童群体等都存在客观差异, 因此表5所示指 标框架对中国城市社区环境的适用性有待进行进一步的实证判断和修 订。例如，“机动车交通环境” 中的 “紧急停靠车道或空间”、“步 行/骑行环境” 中的 “路肩” “求助装置” 等在中国城市环境中并不常

\subsection{Evaluation Indicator Framework of the Community} Environment for Children's Mobility Safety

Based on English literature review, an evaluation indicator system of the community environment for children's mobility safety is established as shown in Table 5, consisting of 3 firstlevel indicators, 11 second-level indicators, 29 third-level indicators, and more fourth-level indicators. As the first-level indicators, motor traffic environment and walking / bicycling environment mainly assess children's mobility safety in terms of motor traffic control, walking / bicycling travel supporting, travel support at crossings, good sight and signs, and other guarantees; other indicators range from social safety, perceptive safety, physical health to safety awareness.

\subsection{Limitations of Indicators from English Literature and Their Adaption to China's Reality}

From the above, it could be concluded that studies on evaluation indicators for children's mobility safety are still insufficient. This study retrieved English literature with four types of keywords and found a convergent trend in the research interests. For instance, existing safety studies on children's activity spaces mainly focus on surveillance and protection by sights, traffic connectivity, and safety factors of local environment (such as lighting) with less considerations on the continuity of children's activities ${ }^{(3}$. Although several guidelines (e.g., Street Design Manual and Making Safer Streets ${ }^{[51]}$ in New York City) have been published, and multiform childrenoriented projects have been implemented (such as the Greenlight Project in Port Philip, Melbourne City, Australia, We Go to School Alone in Italy, and the Walking School Bus programs in the United States, Australia, and New Zealand ${ }^{[52][53]}$, none of them have integrated with existing knowledge on diverse safety factors systematically, and little evaluation has been made on their reliability and effectiveness.

The literature articles and the assessment tools discussed above, as well as their cultural backgrounds, situation of communities, and target group (children), are not based on in China's reality. Therefore, the applicability of the indicator framework in Table 5 to Chinese urban community environment needs further verification and adaption. For instance, "emergency stop lane or space" of the "motor traffic environment," and "road shoulders" and "help-seeking devices" under the "walking / bicycling environment" are not common in Chinese cities and could be removed in practice; The land use pattern, planning approaches, and building layout of Chinese urban communities
3) 随着儿童的成长, 其活 动半径会逐渐扩大。 为了保证儿童随着年龄 增长可逐渐实现独立出 行, 适合儿童活动的答 空间占之间应存在连续 空间点之间应存在连续 的安全路径。

(3) Children's activity radius gradually expands as they grow up, which requires high continuity of safe paths between various spaces to ensure CIM. 
表5: 社区环境儿童出行安全评价指标框架

Table 5: The evaluation indicator framework of children's mobility safety in the community environmen

\begin{tabular}{|c|c|c|c|}
\hline $\begin{array}{l}\text { 一级指标 } \\
\text { First-level indicators } \\
\qquad(\mathrm{N}=3)\end{array}$ & $\begin{array}{c}\text { 二级指标 } \\
\text { Second-level indicators } \\
(\mathbf{N}=11)\end{array}$ & $\begin{array}{c}\text { 三级指标 } \\
\text { Third-level indicators } \\
(\mathbf{N}=29)\end{array}$ & $\begin{array}{l}\text { 四级指标 } \\
\text { Fourth-level indicators }\end{array}$ \\
\hline \multirow{7}{*}{$\begin{array}{l}\text { 机动车交通环境 } \\
\text { Motor traffic } \\
\text { environment }\end{array}$} & \multirow[t]{2}{*}{$\begin{array}{l}\text { 交通控制 } \\
\text { Traffic control }\end{array}$} & $\begin{array}{l}\text { 速度控制 } \\
\text { Speed control }\end{array}$ & $\begin{array}{l}\text { 减速带、减速道、特殊速度区域、环形交叉口 } \\
\text { Speed bumps, ramps, special speed zones, roundabout }\end{array}$ \\
\hline & & $\begin{array}{l}\text { 交通量a }^{a} \\
\text { Traffic volume }\end{array}$ & $\begin{array}{l}\text { 车道宽度、数量、通行率, 年平均日交通量 } \\
\text { Width, amount, and passing rate of lanes; annual average daily traffic (AADT) }\end{array}$ \\
\hline & \multirow[t]{3}{*}{$\begin{array}{l}\text { 视觉保障及提示 } \\
\text { Good sight and signs }\end{array}$} & $\begin{array}{l}\text { 标识ab } \\
\text { Signs }\end{array}$ & $\begin{array}{l}\text { 车道划分线, 停止、速度、方向、车道、行人穿行等提示 } \\
\text { Lane dividing lines; signs of stop, speed, direction, lanes, pedestrian passing through, etc. }\end{array}$ \\
\hline & & $\begin{array}{l}\text { 照明ab } \\
\text { Lighting }\end{array}$ & $\begin{array}{l}\text { 亮灯率、照度 } \\
\text { Light working ratio, illumination }\end{array}$ \\
\hline & & $\begin{array}{l}\text { 车道弯曲度 }^{b} \\
\text { Road curvature }\end{array}$ & $\begin{array}{l}\text { 是否影响视线、速度控制、转弯危险等 } \\
\text { Affect vision or not, speed control, turning danger }\end{array}$ \\
\hline & \multirow[t]{2}{*}{$\begin{array}{l}\text { 其他保障 } \\
\text { Other guarantees }\end{array}$} & $\begin{array}{l}\text { 坚向设计 }{ }^{\mathrm{b}} \\
\text { Vertical design }^{\mathrm{b}}\end{array}$ & $\begin{array}{l}\text { 排水设施 } \\
\text { Drainage facilities }\end{array}$ \\
\hline & & $\begin{array}{l}\text { 紧急停靠车道或空间 } \\
\text { Emergency stop lane or space }^{b}\end{array}$ & - \\
\hline \multirow{16}{*}{$\begin{array}{l}\text { 步行/骑行环境 } \\
\text { Walking / bicycling } \\
\text { environment }\end{array}$} & \multirow[t]{14}{*}{$\begin{array}{l}\text { 通行保障 } \\
\text { Travel supporting }\end{array}$} & \multirow[t]{6}{*}{$\begin{array}{l}\text { 通行空间基本特征 } \\
\text { Basic characteristics of travel space }\end{array}$} & $\begin{array}{l}\text { 数量ab } \\
\text { Quantity }{ }^{\mathrm{ab}}\end{array}$ \\
\hline & & & $\begin{array}{l}\text { 坡度 } \\
\text { Slope }^{\mathrm{ab}}\end{array}$ \\
\hline & & & $\begin{array}{l}\text { 宽度 }^{a} \\
\text { Width }^{\mathrm{a}}\end{array}$ \\
\hline & & & $\begin{array}{l}\text { 密度 }^{\mathrm{a}} \\
\text { Density }^{\mathrm{a}}\end{array}$ \\
\hline & & & $\begin{array}{l}\text { 长度ab (如连续度: 是否有尽端路等 ) } \\
\text { Length }^{\text {ab }} \text { (e.g., continuity / connectivity: cul-de-sacs or not) }\end{array}$ \\
\hline & & & 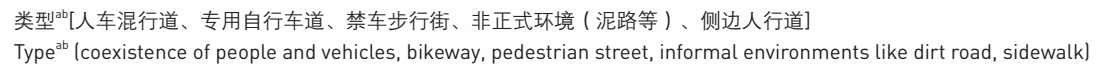 \\
\hline & & \multirow[t]{3}{*}{$\begin{array}{l}\text { 表面 } \\
\text { Surfaces }\end{array}$} & $\begin{array}{l}\text { 铺装材质ab ( 防滑路面、透水路面、盲道 ) } \\
\text { Pavement materials } s^{\text {ab }} \text { (anti-slippery, permeable, sidewalk for the blind) }\end{array}$ \\
\hline & & & $\begin{array}{l}\text { 维护情况 ( } \text { (破损、缺失、裂㖓等) } \\
\text { Maintenance } \text { (damaged, missing, cracks, holes, etc.) }\end{array}$ \\
\hline & & & $\begin{array}{l}\text { 排水系统礼 ( 是否容易积水 ) } \\
\text { Drainage }\end{array}$ \\
\hline & & $\begin{array}{l}\text { 路缘ab } \\
\text { Curb }^{\mathrm{ab}}\end{array}$ & $\begin{array}{l}\text { 形式 (延伸 )、高度、材质、维护情况 } \\
\text { Type (extension), height, materials, maintenance }\end{array}$ \\
\hline & & $\begin{array}{l}\text { 路肩 }^{\mathrm{b}} \\
\text { Road shoulder }\end{array}$ & $\begin{array}{l}\text { 躲避、穿行 } \\
\text { Dodge, crossing }\end{array}$ \\
\hline & & $\begin{array}{l}\text { 隔离缓冲带ab } \\
\text { Isolation or buffer zone }{ }^{\text {ab }}\end{array}$ & $\begin{array}{l}\text { 高度、宽度、形式 (硬质: 栏杆、树等; 软质: 绿化等 ) } \\
\text { Height, width, type (hard: fence, trees, etc.; soft: greening, etc.) }\end{array}$ \\
\hline & & \multirow[t]{2}{*}{$\begin{array}{l}\text { 障碍物 }^{\mathrm{b}} \\
\text { Obstacles }^{\mathrm{b}}\end{array}$} & $\begin{array}{l}\text { 永久性 (杆柱、台阶、花坛、树及树池、固定座椅、垃圾桶等 ) } \\
\text { Permanent (poles, steps, flower terraces, trees and planters, fixed seats, garbage cans) }\end{array}$ \\
\hline & & & $\begin{array}{l}\text { 临时性 ( 停放的机动车、碎玻璃、坑洞、临时桌椅、修缢工事 ) } \\
\text { Temporary (motor vehicles parked, broken glass, cracks and holes, temporary tables and chairs, facilities under repair) }\end{array}$ \\
\hline & \multirow[t]{2}{*}{$\begin{array}{l}\text { 交通节点穿行保障 } \\
\text { Travel supporting at the crossing }\end{array}$} & $\begin{array}{l}\text { 穿行空间 } \\
\text { Crossing } \\
\text { spaces }\end{array}$ & $\begin{array}{l}\text { 过街人行横道、安全岛、过街天桥/地下通道 } \\
\text { Crosswalk, refuge island, overpass / underpass }\end{array}$ \\
\hline & & $\begin{array}{l}\text { 穿行控制 }^{\mathrm{b}} \\
\text { Crossing control }^{\mathrm{b}}\end{array}$ & $\begin{array}{l}\text { 交通信号控制 (视觉、听觉 ) } \\
\text { Traffic control signals (visual and acoustic) }\end{array}$ \\
\hline
\end{tabular}


表5: 社区环境儿童出行安全评价指标框架

Table 5: The evaluation indicator framework of children's mobility safety in the community environment

\begin{tabular}{|c|c|c|c|}
\hline $\begin{array}{l}\text { 一级指标 } \\
\text { First-level indicators } \\
\qquad(\mathrm{N}=3)\end{array}$ & $\begin{array}{c}\text { 二级指标 } \\
\text { Second-level indicators } \\
(\mathrm{N}=11)\end{array}$ & $\begin{array}{c}\text { 三级指标 } \\
\text { Third-level indicators } \\
(\mathbf{N}=29)\end{array}$ & $\begin{array}{l}\text { 四级指标 } \\
\text { Fourth-level indicators }\end{array}$ \\
\hline \multirow{8}{*}{$\begin{array}{l}\text { 步行/骑行环境 } \\
\text { Walking / bicycling } \\
\text { environment }\end{array}$} & \multirow[t]{2}{*}{$\begin{array}{l}\text { 交通节点穿行保障 } \\
\text { Travel supporting at the crossing }\end{array}$} & $\begin{array}{l}\text { 路缘 }{ }^{\mathrm{B}} \\
\mathrm{Curb}^{\mathrm{b}}\end{array}$ & $\begin{array}{l}\text { 形式 ( 延伸 ) } \\
\text { Type (extension) }\end{array}$ \\
\hline & & $\begin{array}{l}\text { 标识/铺装 } \\
\text { Signs / paving }\end{array}$ & $\begin{array}{l}\text { 斑马线、行为提醒标识、儿童易懂的过街标识 } \\
\text { Zebra crossings, behavior reminers, crossing signs for children }\end{array}$ \\
\hline & \multirow[t]{3}{*}{$\begin{array}{l}\text { 视觉保障及提示 } \\
\text { Good sight and signs }\end{array}$} & $\begin{array}{l}\text { 标识 }^{b} \\
\text { Signs }^{b}\end{array}$ & $\begin{array}{l}\text { 警示、方向、规则、步道状况 } \\
\text { Warning, direction, regulation, sidewalk conditions }\end{array}$ \\
\hline & & $\begin{array}{l}\text { 照明 } \\
\text { Lighting }^{\text {ab }}\end{array}$ & $\begin{array}{l}\text { 亮灯率、照度、行人尺度、位置 } \\
\text { Light working ratio, illumination, pedestrian scale, location }\end{array}$ \\
\hline & & $\begin{array}{l}\text { 视线 }^{b} \\
\text { Sight }^{b}\end{array}$ & $\begin{array}{l}\text { 视线可达机动车道以及其他道路使用者 (行人、骑行者 ) } \\
\text { Sight to motorways and other pedestrians and bicycle riders }\end{array}$ \\
\hline & \multirow[t]{3}{*}{$\begin{array}{l}\text { 其他保障 } \\
\text { Other guarantees }\end{array}$} & \multirow[t]{2}{*}{$\begin{array}{l}\text { 周边监视 } \\
\text { Surrounding surveillance }\end{array}$} & $\begin{array}{l}\text { 被动监视 }{ }^{\mathrm{ab}} \text { ( 来自建筑物内及其他行人的视线) } \\
\text { Passive surveillance }\end{array}$ \\
\hline & & & $\begin{array}{l}\text { 主动监视 }{ }^{b} \text { ( 摄像头等监控设备, 警察、安全巡视志愿者 ) } \\
\text { Active surveillance }\end{array}$ \\
\hline & & $\begin{array}{l}\text { 求助装置 }^{b} \\
\text { Help-seeking devices }\end{array}$ & $\begin{array}{l}\text { 报警装置、电话 } \\
\text { Alarm devices, telephones }\end{array}$ \\
\hline \multirow[t]{7}{*}{$\begin{array}{l}\text { 其他指标 } \\
\text { Other indicators }\end{array}$} & $\begin{array}{l}\text { 社会安全 } \\
\text { Social safety }\end{array}$ & $\begin{array}{l}\text { 犯罪与威胁控制 }^{\text {Cb }} \\
\text { Crime and threat control }{ }^{\mathrm{ab}}\end{array}$ & $\begin{array}{l}\text { 犯罪率与暴力行为: 攻击、骚扰、欺凌、反社会行为 } \\
\text { Crime rate and behaviors: attack, harassment, bullying, antisocial behavior }\end{array}$ \\
\hline & \multirow[t]{4}{*}{ 感知安全 } & $\begin{array}{l}\text { 邻里关系 } \\
\text { Neighborhood relationship }\end{array}$ & $\begin{array}{l}\text { 信任度、熟知度 } \\
\text { Trust, close-knit }\end{array}$ \\
\hline & & $\begin{array}{l}\text { 特殊环境与暗示 }^{\mathrm{b}} \\
\text { Special environments and hints }\end{array}$ & $\begin{array}{l}\text { 交叉路口、机动车道交界处, 涂鸦、恶意破坏、废弃建筑物 } \\
\text { Crossroads, junction to motorws; grafiti, vandalism, abandoned buildings }\end{array}$ \\
\hline & & $\begin{array}{l}\text { 邻里识别度 } \\
\text { Legibility within the neighborhood }\end{array}$ & $\begin{array}{l}\text { 易于寻路 } \\
\text { Easy for wayfinding }\end{array}$ \\
\hline & & $\begin{array}{l}\text { 其他因素 } \\
\text { Other factors }\end{array}$ & $\begin{array}{l}\text { 陌生人、无人看管的动物 (狗等) } \\
\text { Strangers, unwatched animals (dog etc. }{ }^{b}\end{array}$ \\
\hline & $\begin{array}{l}\text { 生理健康 } \\
\text { Physical health }\end{array}$ & $\begin{array}{l}\text { 空气质量与声环境 } \\
\text { Air quality and acoustic environment }\end{array}$ & $\begin{array}{l}\text { 机动车尾气排放、难闻的气味、噪音 } \\
\text { Vehicle exhaust, odor, noise }\end{array}$ \\
\hline & $\begin{array}{l}\text { 安全意识 } \\
\text { Safety awareness }\end{array}$ & $\begin{array}{l}\text { 对安全的重视度 } \\
\text { Attention to safety }\end{array}$ & $\begin{array}{l}\text { 区域 (社区) 对道路安全的重视程度 } \\
\text { The attention of an area (community) to road safety }\end{array}$ \\
\hline
\end{tabular}

注

1. a表示原文献中明确指出可量化的指标; b 表示以 “有/无” 属性评价的指标; 无标记表示原文献未提及如何评价该指标。

2. 18项评价工具中有两项（SPACES与WABSA）涉及骑行安全，其中前者未明确区分步行与骑行环境评估指标，后者虽将 骑行类指标单独列出，但与其他评价工具中的步行类指标基本完全重合，包括机动车交通量（AADT），机动车数量与迹 度、最外侧机动车道宽度、自行车道或路肩宽度、路缘、雨水排放格栅, 以及转弯视线等。故本表将步行与骑行环境的评 价指标归为一类一级指标。
NOTES

1. The quantitative indicators in the 18 assessment tools are marked with "a;" the qualitative ones that can be only described with "yes / no" are marked with "b;" and those without any mark means that the evaluation methods are not mentioned in the literature.

2. Bicycling safety is only mentioned in SPACES and WABSA among the 18 tools. SPACES does not distinguish indicators for bicycling environment from walking environment; Although WABSA lists bicycling indicators separately, these indicators are almost same with the walking ones in the other tools (such as AADT, amount and speed of vehicles, width of the outermost motorway, bicycle lanes or road shoulders, curbs, rainwater grates, and sight at turns). Therefore, in this table the indicators for these two environments are merged into one first-level category. 
(4) 如2018年大众汽车集 团 (中国) 与中国妇女 发展基金会共同启动的 “儿童交通友好社区” 项目

4) Such as the Children Mobility Friendly Community colaunched by the Volkswagen Group (China) and the China Women's Development Foundation in 2018
见，因此在实际使用中应酶情剔除; 中国城市居住区的用地现状、规 划方法和住宅平面布局往往使得 “被动监视” 难以形成; 对以社区为 单位的 “机动车交通量” 的统计有赖于提前建立完善的数据库; “临 时性障碍物” 属于可变化的因子, 作为评价指标具有较大不确定性, 可根据社区实际情况选择性使用。其余指标与中国城市社区环境特征 较为相符, 适合作为儿童出行安全评价指标。

在提升社区环境、促进出行安全方面, 当前中国的政策文件以地 方政府制定的指导性规范为主, 如香港于 2000 年启动的 “行人环境改 善计划”、上海市于2016年发布的《街道设计导则》, 以及广东省于 2017年发布的《步行与自行车交通蓝皮书》 ${ }^{[54}$ 等, 这些文件将安全作为 目标导向之一, 表明了政府层面对全域龄群体出行环境安全的重视, 但在科学的出行安全评价指标体系出现之前, 其可靠性及全面性均有 待评估。除此之外, 相关项目实践也仍处于探索阶段。因此, 本研究 基于国外文献梳理出的儿童出行安全评价指标框架, 将有助于对现有 出行环境建设规范中出行安全的相关要求进行系统性完善、建立更为 精细化的分层指标体系, 为相关实践项目中的各阶段评估应用提供有 益参考。LAF make it difficult to form "passive surveillance;" The assessment of "motor traffic volume" in the community requires a sound built-up database; "Temporary obstacles" are changeable with great uncertainties, which should be an optional indicator. Other indicators are suitable for evaluating the community environment in Chinese cities for children's mobility safety.

So far, local governments in China have formulated a series of guidelines to enhance mobility safety by improving the community environment, such as the Pedestrian Environment Improvement Scheme launched in Hong Kong since 2000, the Shanghai Street Design Guidelines in 2016, and the Walking and Bicycling Transportation Bluepaper issued by Guangdong Province in $2017^{[54]}$. Taking safety as one of the major goals, these guidelines showcase the government's attention to the mobility safety of urban environment for all age groups. However, their reliability and comprehensiveness require further examination with a scientific indicator system, and related practices ${ }^{(4)}$ are still in the exploratory phase. Therefore, the evaluation indicator framework for children's mobility safety proposed in this study can help systematically improve the mobility safety requirements of the existing regulations and guidelines to establish a multi-leveled indicator system and provide reference for performance evaluation practice at all stages. LAF 
[1] Qiu, B. (2007). China's paradigm in the third urbanization wave. City Planning Review, 31(6), 9-15.

[2] China Young Pioneers National Working Commission, \& China Youth and Children Research Center. (2007, Novemberl. The name of the children is today - the blue book of the development of contemporary Chinese blue book of the develop cht of contering and Media

3] UNICEF. (2018). Child Friendly Cities Initiative Handbook. Retrieved from https://www.unicef.org/media/56291/file/ \%E5\%84\%BF\%E7\%AB\%A5\%E5\%8F\%8B\%E5\%A5\%BD\%E 5\%9Е\%8B\%Е5\%9F\%8Е\%Е5\%B8\%82\%Е8\%A7\%84\%E5\%8 $8 \% 92 \% E 6 \% 89 \% 8 B \%$ E5 $\% 86 \% 8 C$. pdf

[4] UNICEF. (2018, May). Shaping urbanization for children: A handbook on child-responsive urban planning. Retrieved from https://www.unicef.org/publications/files/UNICEF Shaping_urbanization_for_children_handbook_2018.pdf

[5] UNICEF. (2018, April). Child Friendly Cities and Communities Handbook. Retrieved from https://www. unicef.org/eap/reports/child-friendly-cities-andcommunities-handbook

[6] UNICEF Innocenti Research Center, International Secretariat for Child Friendly Cities. (2004, March 15). Building Child Friendly Cities: A Framework for Action. Retrieved from https://www.unicef-irc.org/ publications/416-building-child-friendly-cities-aframework-for-action.html

[7] UNICEF. (n. d.). Child Friendly Community SelfAssessment Tool for Children. Retrieved from https:// s25924.pcdn.co/wp-content/uploads/2017/11/SelfAssessment-Tool-for-Children.pdf

[8] Cruise, S., Hunter, R. F., Kee. F., Donnelly, M., Ellis, G., \& Tully, M. A. (2017). A comparison of road- and footpathbased walkability indices and their associations with active travel. Journal of Transport and Health, (6), 119127. doi:10.1016/j.jth. 2017.05.364

[9] Bödeker, M., Finne, E., Kerr, J., \& Bucksch, J. (2018). Active travel despite motorcar access. A city-wide, GISbased multilevel study on neighborhood walkability and active travel in Germany. Journal of Transport and Health, active travel in Germany. Journal of 18 . doi:10.1016/j.jth.2018.03.009

[10] Millington, C., Thompson, C. W., Rowe. D. A., Aspinall, P., Fitzsimons, C., Nelson, N. M., \& Mutrie, N. (2009). Development of the Scottish Walkability Assessment Tool (SWAT). Health and Place, 15(2), 474-481. doi:10.1016/ j.healthplace.2008.09.007

[11] Rosenberg, D. E., Ding, D., Sallis, J. F., Kerr, J., Norman, G. J., Durant, N., ... Saelens, B. E. (2009). Neighborhood Environment Walkability Scale for Youth (NEWS-Y): Reliability and Relationship with Physical Activity. Preventive Medicine, 49(2), 213-218. doi:10.1016/ j.ypmed.2009.07.011

[12] Pikora, T., Bull, F., Jamrozik. K., Knuiman, M., Gilescorti, B., \& Donovan, R. J. (2002). Developing a reliable audit instrument to measure the physical environment for physical activity. American Journal of Preventive Medicine, 23(3), 187-194. doi:10.1016/507493797(02)00498-1
[13] Sallis, J. F., Cain, K. L., Conway. T. L., Gavand, K. L., Millstein, R. A., Geremia, C. M., ... King, A. C. (2015). Is You Neighborhood Designed to Support Physical Activity? A Brief Streetscape Audit Tool. Preventing Chronic Disease, 12(9), 1-11. doi:10.5888/pcd12.150098

[14] Emery, J., Crump, C., \& Bors, P. (1998). Walking and Bicycling Suitability Assessment. Retrieved from http:// wabsa.web.unc.edu/

[15] Troped, P. J., Cromley, E. K., Fragala, M. S., Melly, S. J Hasbrouch, H. H., Gortmaker, S. L., \& Brownson, R. C. (2006). Development and Reliability and Validity Testing of an Audit Tool for Trail/Path Characteristics: The Path Environment Audit Tool (PEAT). Journal of Physical Activity and Health, 3(S1), S158-S175. doi:10.1123/jpah.3.s1.s158

[16] Clifton, K. J., Smith, A. L., \& Rodriguez, D. A. (2007). The Development and Testing of an Audit for the Pedestrian Environment. Landscape and Urban Planning, 80(1), $95-$ 110. doi:10.1016/j.landurbplan.2006.06.008

[17] Williams, J. E., Evans, M., Kirtland, K. A., Cavnar, M. M., Sharpe, P. A., Neet, M. J., ... Cook, A. (2005). Development and Use of a Tool for Assessing Sidewalk Maintenance as an Environmental Support of Physical Activity. Health Promotion Practice, 6(1), 81-88. doi:10.1177/1524839903260595

[18] Hoehner, C. M., Ivy, A., Brennan Ramirez, L. K., Handy, S. L. \& Brownson, R. C. (2007). Active Neighborhood Checklist: A User-Friendly and Reliable Tool for Assessing ActivityFriendliness. American Journal of Health Promotion, 21(6) 534-537. doi:10.4278/0890-1171-21.6.534

[19] Kaczynski, A. T., Wilhelm Stanis, S. A., Besenyi, G. M. (2012) Development and Testing of a Community Stakeholder Park Audit Tool. American Journal of Preventive Medicine, 42(3), 242-249. doi:10.1016/j.amepre.2011.10.018

[20] Saelens, B. E., Frank, L. D., Auffrey, C., Whitaker, R. C., Burdette, H. L., \& Colabianchi, N. (2006). Measuring Physical Environments of Parks and Playgrounds: EAPRS Instrument Development and Inter-Rater Reliability. Journal of Physical Activity and Health, 3(s1), 190-207. doi:10.1123/jpah.3.s1.s190

[21] Saelens, B. E. (2006, July). Environmental Assessment of Public Recreation Spaces (EAPRS) Tool. Retrieved from https://www.activelivingresearch.org/environmentalassessment-public-recreation-spaces-eaprs-tool

[22] Peltola, H., Rajamäki, R., \& Luoma, J. (2013). A tool for safety evaluations of road improvements. Accident Analysis and Prevention, (60), 277-288. doi:10.1016/ j.aap. 2013.04 .008

[23] Freeman, L., Neckerman, K. M., Schwartz-Soicher, O., Quinn, J. W., Richards, C., Bader, M. D. M., ... Rundle, A. (2013). Neighborhood walkability and active travel (walking and cycling) in New York City. Journal of Urban Healthbulletin of The New York Academy of Medicine, 90(4), 575585. doi:10.1007/s11524-012-9758-7

[24] Saelens, B. E., Sallis, J. F., \& Frank, L. D. (2003). Environmental correlates of walking and cycling: Findings from the transportation, urban design, and planning literatures. Annals of Behavioral Medicine, 25(2), 80-91. doi:10.1207/S15324796ABM2502_03
[25] Buck, C., Tkaczick, T., Pitsiladis, Y. P., De Bourdehaudhuij, I., Reisch, L. A., Ahrens, W., \& Pigeot, I. (2014). Objective measures of the built environment and physical activity in children: From walkability to moveability. Journal of Urban Health-bulletin of The New York Academy of Medicine, 92(1), 24-38. doi:10.1007/s11524-014-9915-2

[26] Bird, M. E. Datta, G. D., Van Hulst, A., Kestens, Y., \& Barnett, T. A. (2015). A reliability assessment of a directobservation park evaluation tool: The Parks, Activity an Recreation among Kids (PARK) tool. BMC Public Health, 15(1), 906-916. doi:10.1186/s12889-015-2209-0

[27] Rigolon, A., \& Németh, J. (2018). A QUality INdex of Parks for Youth (QUINPY): Evaluating urban parks through geographic information systems. Environment and Planning B: Planning and Design, 45(2), 275-294. doi:10.1177/0265813516672212

[28] Jenkins, G., Yuen, H. K., Rose, E. J., Maher, A. I., Gregory, K. C., \& Cotton, M. E. (2015). Disparities in Quality of Park Play Spaces between Two Cities with Diverse Income and Race/Ethnicity Composition: A Pilot Study. International Journal of Environmental Research and Public Health, 12(7), 8009-8022. doi:10.3390/ijerph120708009

[29] Orso, G., \& Migliore, M. (2020). A GIS-based method for evaluating the walkability of a pedestrian environment and prioritised investments. Journal of Transport Geography, (82), 102555. doi:10.1016/j.jtrangeo.2019.102555

[30] Janssen, l., Ferrao, T., \& King, N. (2016). Individual, family, and neighborhood correlates of independent mobility among 7 to 11 -year-olds. Preventive Medicine Reports, (23), 98-102. doi:10.1016/j.pmedr.2015.12.008

[31] Carver, A., Panter, J., Jones, A., \& Van Sluijs, E. M. F. (2014). Independent mobility on the journey to school: A joint cross-sectional and prospective exploration of social and physical environmental influences. Journal of Transport and Health, 1(1), 25-32. doi:10.1016/ j.jth.2013.12.003

[32] Lin, E., Witten, K. Oliver, M. Carroll, P., Asiasiga, L., Badland, H., \& Parker, K. (2017). Social and builtenvironment factors related to children's independent mobility: The importance of neighbourhood cohesion and connectedness. Health and Place, (46), 107-113. doi:10.1016/j.healthplace.2017.05.002

[33] Kytta, M. (2004). The extent of children's independen mobility and the number of actualized affordances as criteria for child-friendly environments. Journal of Environmental Psychology, 24(2), 179-198. doi:10.1016/ S0272-4944(03)00073-2

[34] Ghekiere, A., Deforche, B., Carver, A., Mertens, L., De Geus, B., Clarys, P., ... Van Cauwenberg, J. (2017). Insights into children's independent mobility for transportation cycling: Which socio-ecological factors matter? Journal of Science and Medicine in Sport, 20(3), 267-272. doi:10.1016/j.jsams.2016.08.002

[35] Sharmin, S., \& Kamruzzaman, M. (2017). Association between the built environment and children's independent mobility: A meta-analytic review. Journal of Transport Geography, (61), 104-117. doi:10.1016/ j.jtrangeo.2017.04.004 
[36] Gilbert, H., Whitzman, C., \& Pieters, J., \& Allan, A. (2018). Children's everyday freedoms: Local government policies o children and sustainable mobility in two Australian states. Journal of Transport Geography, (71), 116-129. doi:10.1016/ j.jtrangeo.2018.07.007

[37] Buliung, R., Larsen, K., Faulkner, G., \& Ross, T. (2017). Children's independent mobility in the City of Toronto Canada. Travel Behaviour and Society, (9), 58-69. doi:10.1016/j.tbs.2017.06.001

[38] Stark, J., Frühwirth, J., \& Aschauer, F. (2018). Exploring independent and active mobility in primary school children in Vienna. Journal of Transport Geography, (68), 31-41. doi:10.1016/j.jtrangeo.2018.02.007

[39] Cordovil, R., Lopes, F., \& Neto. C. (2015). Children's (in) dependent mobility in Portugal. Journal of Science and Medicine in Sport, 18(3), 299-303. doi:10.1016/ j.jsams.2014.04.013

[40] Bhosale, J., Duncan, S., \& Schofield, G. (2017). Intergenerational change in children's independent mobility and active transport in New Zealand children and parents. Journal of Transport and Health, (7), 247-255. doi:10.1016/ j.jth.2017.09.004

[41] Ruiz-Padillo, A., Pasqual, F. M., Uriarte, A. M. L., \& Cybis, H. B. B. (2018). Application of multi-criteria decision analysis methods for assessing walkability: A case study in Porto Alegre, Brazil. Transportation Research Part D: Transport and Environment, (63), 855-871. doi:10.1016/j.trd.2018.07.016

[42] Schoeppe, S., Duncan, M. J., Badland, H., Oliver, M., \& Curtis, C. (2013). Associations of children's independent mobility and active travel with physical activity, sedentary behaviour and weight status: A systematic review. Journal of Science and Medicine in Sport, 16(4), 312-319. doi:10.1016/ j.jsams.2012.11.001

[43] Veitch, J., Carver, A., Salmon, J., Abbott, G., Ball, K.

Crawford, D., ... Timperio, A. (2017). What predicts children's active transport and independent mobility in disadvantaged active transport and inde. jeighborhoods? Heath and Place, (44), 103-109. doi:10.101 t/ j.healthplace.2017.02.003

[44] Crawford, S., Bennetts, S. K., Hackworth, N. J., Green, J., Graesser, H., Cooklin, A., ... Nicholson, J. M. (2017). Worries, weirdos', neighborhoods and knowing people: a qualitative study with children and parents regarding children's independent mobility. Health and Place, (45), 131-139. doi:10.1016/j. healthplace.2017.03.005

[45] Giles-Corti, B., Wood, G., Pikora, T., Learnihan, V., Bulsara, M., Van Niel, K. P., ... Villanueva, K. (2011). School site and the potential to walk to school: The impact of street connectivity and traffic exposure in school neighborhoods. Health and Place, 17(2), 545-550. doi:10.1016/j.healthplace.2010.12.01

[46] Hume, C., Timperio, A., Salmon, J., Carver, A., Gilescorti, B., \& Crawford, D. (2009). Walking and Cycling to School Predictors of Increases among Children and Adolescents. Predictors of Licreas a Amit10.1016/jan or 2008.10011 10.01

[47] Battista, G. A., \& Manaugh, K. (2019). Generating walkability from pedestrians' perspectives using a qualitative GIS method. Travel Behaviour and Society, (17), 1-7. doi:10.1016/ j.tbs.2019.05.009
[48] Leden, L., Garder, P., Schirokoff, A., Monterdeibort, H. Johansson, C., \& Basbas, S. (2014). A sustainable city environment through child safety and mobility: A challenge based on ITS? Accident Analysis and Prevention, (62), 406414. doi:10.1016/j.aap.2013.06.013

[49] Gitelman, V., Levi, S., Carmel, R., Korchatov, A., \& Hakkert, S. (2019). Exploring patterns of child pedestrian behaviors at urban intersections. Accident Analysis and Prevention, (122). 36-47. doi:10.1016/j.aap.2018.09.031

[50] Poku-Boansi, M., Amoako, C., \& Atuah, D. O. (2019). Urban travel patterns and safety among school children around Accra, Ghana. Journal of Transport and Health, (15), 100660. doi:10.1016/j.jth. 2019.100660

[51] Long, Y., \& Chen, Y. (2017). A special issue on street walkability in Shanghai Planning Review. Retrieved from https://www.beijingcitylab.com/projects-1/22-streeturbanism/

[52] Weitzman, C., Chen, Y., \& Luo, Z. (2008). Policies and Practices that Promote Children's Independent Mobility. Urban Planning International, 23(5), 56-61. doi:10.3969/ j.issn. 1673-9493.2008.05.009

[53] Meng, X., Li, L., \& Fu, B. (2019). Progress and Enlightenment of Research on Children's Independent Activity Abroad. Human Geography, 34(4), 20-31.

[54] Natural Resources Defense Council, \& School of Architecture, Tsinghua University. (2019, May). Evaluation of walkability in Chinese Cities - Research on Walkability of Urban Vitality Center. Retrieved from https://www. beijingcitylab.com/projects-1/22-street-urbanism/

\section{《景观设计学》2020年第1期 (总第43期) 更正 \\ Correction of No. 1 Issue, No. 8 Volume of Landscape Architecture Frontiers (No. 43 in total)}

《从人工橡胶林到热带雨林的回归一一西双版纳三达山热带雨林生态修复》一文DOI号更正为:

The DOI of the article "The Rebirth of Tropical Rainforest - Ecological Restoration Planning for Sanda Mountain of Xishuangbanna, China” from page 108 to 125 should be:

https://doi.org/10.15302/J-LAF-1-040012 